\title{
Clinical and subjective outcomes of abdominal mesh surgery (sacrocolpopexy and sacrohysteropexy) for apical prolapse: a single-center experience
}

\author{
MONIKA ANANT ${ }^{1}$, (1) AMRITA SINGH ${ }^{2}$, (1) SHWETA GUPTA ${ }^{3}$, (1) MUKTA AGARWAL ${ }^{1}$, (1) SANGAM KUMARI ${ }^{1}$, \\ (10) ANITA PASWAN ${ }^{1}$
}

1Department of Obstetrics and Gynaecology, All India Institute of Medical Sciences-Patna, Patna, India

2Department of Obstetrics and Gynaecology, Pristyn Care, New Delhi, India

${ }^{3}$ Department of Obstetrics and Gynaecology, Anugrah Narayan Magadh Medical College (ANMCH) , Gaya, India

\begin{abstract}
Objective: Apical compartment prolapse affects the day-to-day activities while decreasing the quality of life of affected women. With better anatomical understanding of the supports of pelvic organs and increasing life expectancy of women, reconstructive pelvic surgeries may be offered to all. Restoration of anatomical positions of displaced pelvic organs can be achieved either by open abdominal, laparoscopic or vaginal suspension procedures.
\end{abstract}

The aim of the study was to assess the perioperative and short term (6-24 months) post-operative success rates, complications and subjective satisfaction of women undergoing apical prolapse surgery by abdominal sacrohysteropexy for uterine prolapse and sacrocolpopexy for vault prolapse.

Materials and Methods: A prospective observational study of 41 patients who underwent abdominal mesh surgery for apical prolapse, during 2016-2018, in a tertiary hospital of eastern India. These patients were followed up for over a 2 years period, to assess the outcome measures.

Results: Mean follow up of patients was 18.3 months with apical success rates (defined as Pelvic Organ Prolapse Quantifications System staging of 0/1 post-surgery) of $100 \%$ (mean point C -6.55 and mean point D - 8.8). Intraoperative complications encountered were bladder injury and hemorrhage. No mesh complications occurred during the study period. A significant reduction in the subjective scores of vaginal symptoms, sexual wellbeing and quality of life was also noted in study participants.

Conclusion: Abdominal sacrocolpopexy and sacrohysteropexy showed excellent anatomical success rates as per results of this study. They provide optimum apical support with a good functional outcome for patients with vaginal apical prolapse.

Keywords: Apical prolapse; sacrocolpopexy; abdominal; mesh

\section{INTRODUCTION}

Pelvic organ prolapse (POP) is the descent of one or more of the pelvic structures (bladder, uterus, bowel) from their normal anatomic location towards or through the vaginal opening. Most often POP requires surgical treatment. Women have an 11 percent chance of undergoing surgery for POP by 80 years of age. ${ }^{1,2}$ Women of all ages may be affected, although pelvic

Address for Correspondence: Monika Anant, Department of Obstetrics Gynaecology, All India Institute of Medical Sciences-Patna, Patna, India E-mail: drmonika.anant@gmail.com ORCID ID: orcid.org/0000-0001-7957-379X

Received: 08 February 2021 Accepted: 15 April 2021

${ }^{\circledR}$ Copyright 2021 by the International Society for Pelviperineology / Pelviperineology published by Galenos Publishing House. 
organ prolapse is more common in older women. The etiology is multifactorial resulting in the loss of pelvic support by either a direct injury to the levator ani muscle forming the pelvic basin or neurologic injury to pudendal nerves during vaginal childbirth. Previous hysterectomy for POP and an increased intra-abdominal pressure from chronic coughing, straining with constipation, or repeated heavy weight lifting are the contributory factors.

Apical compartment prolapse occurs when the vaginal cuff/ apex of hysterectomised patients descends down or out through the vagina. This may or may not be associated with anterior (cystocele, urethrocele) or posterior vaginal wall (enterocoele, rectocele) prolapse. Vaginal vault prolapse is defined as the descent of the vaginal cuff below a point that is $2 \mathrm{~cm}$ less than the total vaginal length above the plane of the hymen by the International Continence Society. ${ }^{3}$ Urinary, anorectal, and coital dysfunction may be associated with the prolapse and can affect a women's quality of life negatively. ${ }^{4}$

Surgical options for vault prolapse are reconstructive and obliterative. Abdominal (sacrocolpopexy) and vaginal [sacrospinous fixation (SSF)] are commonly performed reconstructive procedures. In abdominal sacrocolpopexy (ASCP), whether open (OSCP) or laparoscopic (LSCP), a graft material is attached between the vagina and sacrum, supporting the vagina, thereby restoring pelvic anatomy. ASCP is the most durable operation for advanced POP and serves as the criterion (gold) standard against which other operations are compared. ${ }^{5,6}$ However, data on long-term (5 to 10 years) durability of sacrocolpopexy are limited as most studies have reported short term outcomes.

For younger women with POP wishing to become pregnant again, suspension of the uterus can be done by various routes and to different pelvic structures. Operative procedures are ventral hysteropexy (undersurface of the abdominal wall), transvaginal uterosacral SSF, and laparoscopic uterine suspension by suturing round ligaments to the rectus sheath. Sacrohysteropexy attaches a mesh from anterior and posterior cervix at the level of the isthmus to the anterior longitudinal ligament overlying the sacrum retroperitoneally.

Most of the published literature on sacrocolpopexy and sacrohysteropexy are retrospective studies where objective anatomical and surgical outcomes have been dealt with. ${ }^{7}$ The functional component (vaginal symptoms, sexual wellbeing) of prolapse surgery has often been neglected. This article deals with short term operative and anatomical outcome (6-24 months) of open sacrocolpopexy and sacrohysteropexy along with the subjective outcome of vaginal symptoms and sexual wellbeing of females.

\section{MATERIALS AND METHODS}

A prospective observational study was undertaken at the Department of Obstetrics and Gynaecology of a tertiary level hospital after clearance from Institutional Ethics Committee (IEC) AlIMS PAT/no: 92-2016 date: 22/8/2016). A total of 41 patients of apical POP who underwent abdominal mesh surgery during the study period of July 2016-June 2018, were evaluated for short term (6-24 months) surgical outcomes. Subjective evaluation of their vaginal symptoms, sexual wellbeing and overall quality of life was also carried out at follow up.

Sacrocolpopexy was performed in patients in 32 women presenting with vaginal vault prolapse. Abdominal sacrohysteropexy was performed in nine patients desirous of further childbearing or wishing to retain their uterus in ages less than 35 years. Objective assessment of POP was done using the pelvic organ prolapse quantification (POP-Q) scale at preoperative for baseline and every postoperative visit (6 weeks, $6,12,18$ and 24 months). Subjective assessment of Quality of life (QOL) and postoperative subjective success was assessed using the International Consultation on Incontinence Questionnaire for Vaginal Symptoms (ICIQ-VS) at six months follow up visit.

After routine preoperative work-up and obtaining a written informed consent, patients underwent open abdominal surgical procedures of Sacro-hysteropexy and Sacro-colpopexy. Regional anesthesia and modified lithotomy position for surgery were used. Preoperative antibiotic prophylaxis was given to all patients. Non-absorbable synthetic, porous, monofilament polypropylene mesh was used for the fixation of the vault or cervix to sacrum.

In sacrocolpopexy, the bladder was dissected down anteriorly and the rectum was dissected down posteriorly and 5-6 cm area of the vaginal vault bared. The two short arms of $Y$ shaped mesh was attached to vaginal vault by 2 rows of 3 sutures each. The hysteropexy mesh was attached only posteriorly at the cervical isthmic region over a 3-4 cm area with 3 rows of 2 sutures. Posterior point fixation of the long arm of $\mathrm{Y}$ - mesh was done to the anterior longitudinal ligament overlying the first or second sacral vertebra (S1 or S2) after opening the retroperitoneum between the right ureter and sigmoid colon. Routine closure of the retroperitoneum over the mesh was later carried out in all cases. Concomitant repair of pelvic floor defects, if any, was performed vaginally. Bilateral tubal ligation was performed in cases of sacrohysteropexy where no further childbearing was intended. The routine postoperative care was given and most patients were discharged on the seventh postoperative day after stitch removal. Patients were instructed to avoid strenuous activity, heavyweights lifting, straining on stools and to abstain 
from sexual activity immediately postoperative period (6 weeks). Perioperative and postoperative complications were documented in the predesigned proforma for the study. First, follow-up of patients was done at six weeks and thereafter at $6^{\text {th }}-12^{\text {th }}$ months and at $12^{\text {th }}-24^{\text {th }}$ months post-procedure to assess surgical success and patient satisfaction. For the study, short term success of SCP was defined as Stage 0 or stage 1 apical prolapse on clinical examination (objective). Subjective assessment for vaginal symptoms, sexual wellbeing, and overall quality of life was done at six weeks visit. The questionnaire (ICIQ-VS questionnaire) was filled up at the 6 month follow up visit of all patients. We enquired about vaginal bulge symptoms, soreness, pain, dry sensation of vagina, coitus related problems like effect on sex life and relationship with the partner and any interference in everyday life due to vaginal symptoms. A set of 14 questions with answers scored from 0-10 was used. The questionnaire included questions on dragging pain, soreness, dryness, reduced sensation, too loose or lax, lump or vaginal bulge, need for digitation to pass stools, too tight, vaginal symptoms affecting life, sexual life interfered by vaginal symptoms and partner relationship affected by vaginal symptoms.

\section{Statistical analysis}

All data collected was entered in Microsoft excel sheets and data cleaning done before statistical analysis. Numerical variables i.e., age, body mass index (BMI), parity and days of hospital stay were presented as mean \pm standard deviation. Parity as the median. Categorical variables e.g., factors associated with uterovaginal prolapse, its grade, duration of surgery, blood loss, complications of the operation, the success of the operation and patient satisfaction with the results of the procedure were presented as frequencies and percentages. Statistical analysis was performed by IBM SPSS statistical software v22. The range for study data are presented as Means and median. The difference in postoperative from preoperative POP-Q scoring and subjective outcomes has been calculated using Wilcoxon signed rank test.

\section{RESULTS}

Out of 41 patients with pelvic organ prolapse included in the study, 32 underwent ASCP and nine - abdominal sacrohysteropexy. Preoperative all 41 patients had Stage III or IV POP-Q Stage. The mean age, mean BMI and median parity were comparable in both procedure groups. In the ASCP group, the mean age was 47 years, mean BMI: $23.5 \mathrm{~kg} / \mathrm{m}^{2}$ and median parity - 4 . In the abdominal sacrohysteropexy group, mean age was 29.4 years, mean BMI: $21.4 \mathrm{~kg} / \mathrm{m}^{2}$ and median parity was 2 (Table 1).

Posterior colpoperineorrhaphy was the most commonly performed concomitant procedure while anterior colporrhaphy, tubal ligation, Moschowitz repair were also done. In one patient, a concomitant abdominal hysterectomy was performed, followed by sacrocolpopexy to treat stage 4 pelvic organ prolapse (Table 2).

The mean operative time was 107 minutes including the time required to perform any concomitant procedures with average blood loss of $285 \mathrm{ml}$. Intraoperatively, severe pelvic adhesions were encountered in $14.6 \%$ (6/41) patient's apical prolapse patients, causing increased blood loss. A single intraoperative bladder injury was repaired simultaneously. Intraoperative hemorrhage (1000 $\mathrm{ml})$ due to injury to presacral vessels occurred in one patient requiring two units of blood transfusion (Table 3). In the postoperative period, the cause of concern was fever which occurred in 34\% (14/41) of patients. Most febrile episodes subsided within the first 24 hours, however in four patients lasted for 72 hours. In spite of full aseptic precautions, 6/41 (14.7\%) patients had abdominal wound infection leading to wound dehiscence which required secondary surgical repair and prolonged hospital stay. Postoperative ileus was noted in one patient who responded to conservative management. Most patients had nine days of hospital stay (three days before, and six days after the operation). Six patients $(6 / 41,14.5 \%)$ with wound complications had longer stay in hospital (Table 4).

During the six weeks follow-up (Table 5), five (12\%) patients complained of constipation and were treated satisfactorily by a diet modification. Mild discomfort around the incision line was reported by $14.6 \%$ (6/41) patients. Vault infection was seen in two patients which responded to oral antibiotics. Two patients had recurrent urinary tract infection and 7.3\% (3/41) patients

\begin{tabular}{|l|l|l|}
\hline \multicolumn{3}{|l|}{ Table 1. Patient characteristics } \\
\hline $\begin{array}{l}\text { Patient } \\
\text { demographics }\end{array}$ & $\begin{array}{l}\text { Sacrocolpopexy } \\
\text { group }\end{array}$ & $\begin{array}{l}\text { Sacrohysteropexy } \\
\text { group }\end{array}$ \\
\hline Mean age (years) & $47(42-66)$ & $29.4(26-35)$ \\
\hline Mean BMI $\left(\mathrm{kg} / \mathrm{m}^{2}\right)$ & $23.5(21-27.8)$ & $21.4(19.4-27.2)$ \\
\hline Median parity & 3.5 & 2 \\
\hline BMI: Body mass index & \\
\hline
\end{tabular}

Table 2. Concomitant procedures performed

\begin{tabular}{|l|l|}
\hline Posterior colpoperineorrhaphy & $21(51.2 \%)$ \\
\hline Anterior colporrhaphy & $8(19.5 \%)$ \\
\hline BSO & $8(19.5 \%)$ \\
\hline Tubal ligation & $7(17 \% \%)$ \\
\hline Incisional hernia repair & $4(9.75 \%)$ \\
\hline Moschowitz repair & $2(4.8 \% \%)$ \\
\hline Concomitant TAH, myomectomy, CPT repair & 1 each (2.4\%) \\
\hline $\begin{array}{l}\text { BSO: Bilateral salpingo-oophorectomy; TAH: Total abdominal } \\
\text { hysterectomy; CPT: Current procedural terminology }\end{array}$ \\
\hline
\end{tabular}


reported back pain. At the 6-12 months follow up visit, two complained of dyspareunia and another one had vaginitis. None of the patients developed mesh granuloma, mesh erosion or a

\begin{tabular}{|l|l|}
\hline \multicolumn{2}{|l|}{ Table 3. Intraoperative and postoperative complications } \\
\hline Intra operative complication & $9 / 41(21.9 \%)$ \\
\hline Severe adhesion encountered & $1 / 41(2.4 \%)$ \\
\hline Cystostomy & 0 \\
\hline Bowel/rectal injuries & $1 / 41(2.4 \%)$ \\
\hline Haemorrhage (>1000 ml) & $14(34.14 \%)$ \\
\hline Postoperative complication & $6(14.6 \%)$ \\
\hline Fever (first day) & $1(2.4 \%)$ \\
\hline Wound infection/dehiscence & 0 \\
\hline Ileus & 0 \\
\hline Abdominal wall haematoma & 0 \\
\hline Sepsis & \\
\hline Urinary retention/UTI & \\
\hline UTI: Urinary tract infection &
\end{tabular}

Table 4. Intra-operative measurements and hospital stay of study patients

\begin{tabular}{|l|l|}
\hline Mean blood loss $(\mathrm{ml})$ & $284.7(150-1200 \mathrm{ml})$ \\
\hline Mean operative time $(\mathrm{min})$ & $107(50 \mathrm{~min}-212 \mathrm{~min})$ \\
\hline Hospital stay (days) & 9 (range: $6-30$ days $)$ \\
\hline
\end{tabular}

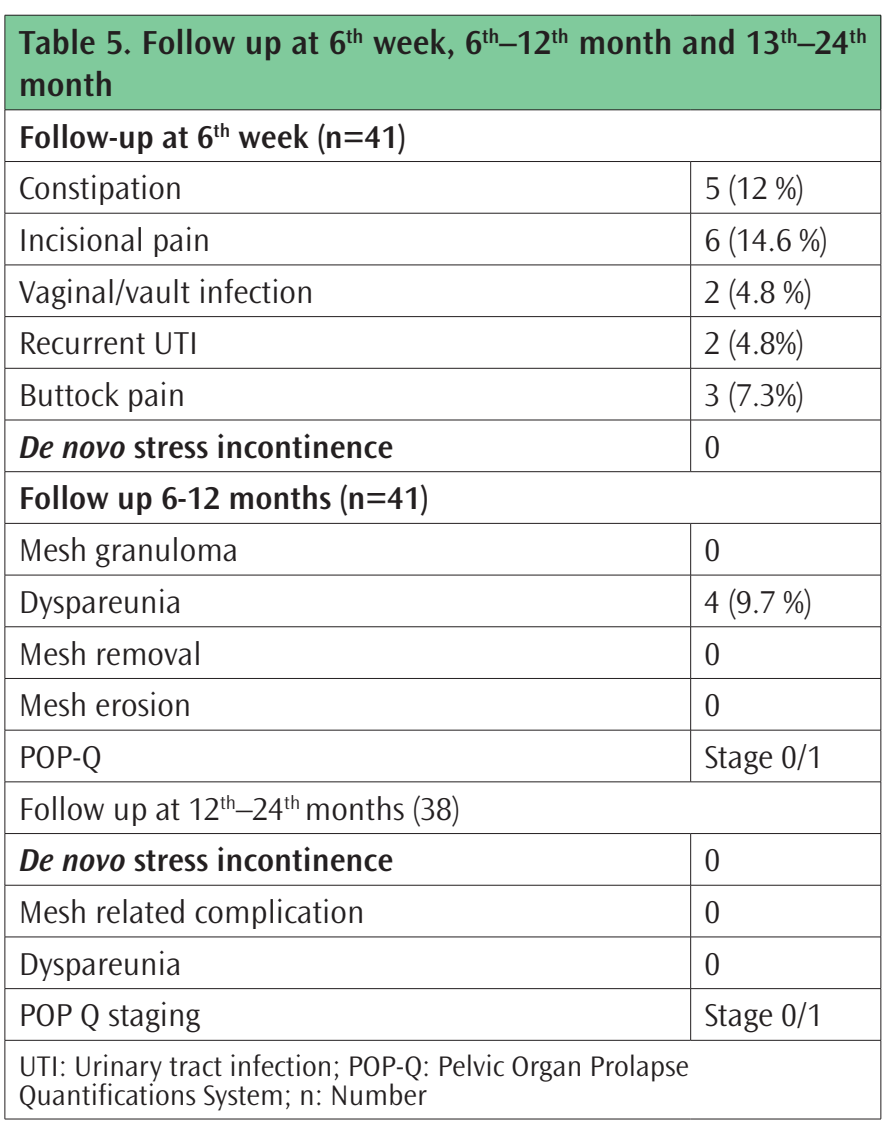

need of mesh removal. No mesh complications, de novo stress incontinence or vaginal bulge symptoms were reported. Patients reporting dyspareunia in the earlier visit were symptomless later. POP-Q staging was determined at each follow-up visit for all patients and the last recorded values were used for analysis. No patient had a prolapse in any compartment post-surgery. (Table 6). One patient who underwent sacrohysteropexy had a feeling of the vaginal bulge, and at examination a POP-Q Stage I anterior compartment prolapse was noted. Apical restoration in all the 41 patients was $100 \%$ (mean point C: -6.55 and mean point D: 8.8). It persisted throughout the follow-up of one year for 22 patients and for two years in 19 patients (Table 7).

In the hysteropexy group of patients $(n=9)$, concomitant sterilization procedure was carried out in seven patients. One hysteropexy patient became pregnant and was delivered by caesarean section at 36 weeks with placenta previa. Her postpartum POP-Q scores remained the same as pre-pregnancy. One nulligravid woman underwent hysteropexy and was advised to undergo follow-up during future pregnancy. Significant subjective improvement was documented by patients in vaginal symptoms, sexual wellbeing and related quality of life, in the questionnaires (Table 8). Most operated patients - 31/35 (85.7\%) experienced improvement in sexual function post-procedure. Four patients were not sexually active pre-procedure.

\section{DISCUSSION}

Pelvic reconstructive procedures aim to correct POP by restoring normal vaginal supports, maintaining urinary and fecal continence, and preserving sexual function by maintaining vaginal capacity and position. ASCP using an open or laparoscopic route and vaginal SSF are the common reconstructive procedures carried out for vaginal vault prolapse. Surgical options for uterus preserving includes ventral hysteropexy (fixation of the uterus to the abdominal wall), transvaginal SSF and laparoscopic uterine suspension by suturing round ligaments to the rectus sheath or cervix to the pectineal ligament.

Age of the patient, presence of comorbidity, previous corrective surgical attempts and the level of post-operative physical and sexual activity desired are the factors that should decide the most appropriate procedure. Surgical expertise of the gynecologist for a particular procedure also influences the operative choices. Lane $^{8}$ in 1962 first described ASCP, where retroperitoneal synthetic, autologous or allograft prosthesis was placed between the vaginal vault and the sacral promontory.

Vaginal SSF, the other commonly performed procedure has a considerable success rate of $69 \%-91 \% .{ }^{9}$ When compared to sacrocolpopexy, SSF has a shorter operative time, lesser 


\begin{tabular}{|c|c|c|c|c|c|c|}
\hline \multicolumn{7}{|c|}{ Preoperative POP-Q measurement $(\mathrm{cm})$} \\
\hline & $\mathrm{Aa}$ & $\mathrm{Ba}$ & $C(n=32)$ & $D(n=9)$ & Ap & Bp \\
\hline Mean & +1.9 & +2.78 & +4.4 & -2 & +0.72 & +0.96 \\
\hline Median & +2 & +4 & +5 & -3 & +2 & +2 \\
\hline Range & -2 to +3 & -2 to +4.5 & +2 to +6.5 & -5 to +8 & -3 to +3 & -3 to +4 \\
\hline \multicolumn{7}{|c|}{ Post-operative POP-Q measurement $(\mathrm{cm})$} \\
\hline & Aa & $\mathrm{Ba}$ & $C(n=32)$ & $D(n=9)$ & Ap & Bp \\
\hline Mean & -2.96 & -2.88 & -6.55 & -8.8 & -2.88 & -2.88 \\
\hline Median & -3 & -3 & -7 & -9 & -3 & -3 \\
\hline Range & -3 to -1 & -3 to -2 & -10 to -5 & -10 to -8 & -3 to -2 & -3 to -2 \\
\hline
\end{tabular}

\section{Table 7. Improved postoperative status by POP-Q scores}

Diff. postoperative to preoperative

\begin{tabular}{|l|l|l|l|l|l|l|}
\hline & Aa & Ba & C & D & Ap & Bp \\
\hline Mean* & $-4.59 *$ & $-5.51^{*}$ & $-11.95^{*}$ & $-10.8^{*}$ & $-3.6^{*}$ & $-3.84^{*}$ \\
\hline *Wilcoxon signed rank test; POP-Q: Pelvic Organ Prolapse Quantifications System & \\
\hline
\end{tabular}

\begin{tabular}{|c|c|c|c|}
\hline \multicolumn{4}{|c|}{ Preoperative ICIQ vs scores } \\
\hline & $\begin{array}{l}\text { VS Score } \\
\left(V_{\max }=53\right)\end{array}$ & $\begin{array}{l}\text { SM Score } \\
\left(\mathrm{SM}_{\max }=58\right)\end{array}$ & $\begin{array}{l}\text { QOL Score } \\
\left(Q 0 L_{\max }=10\right)\end{array}$ \\
\hline Mean & 26.64 & 38.63 & 8.47 \\
\hline Median & 27 & 40 & 9 \\
\hline Range & 12 to 35 & 19 to 58 & 5 to 10 \\
\hline \multicolumn{4}{|c|}{ Postoperative ICIQ vs scores } \\
\hline & VS Score & SM Score & QOL Score \\
\hline Mean & 5.4 & 1.08 & 0.47 \\
\hline Median & 6 & 0 & 0 \\
\hline Range & 2 to 12 & 0 to 2 & 0 to 5 \\
\hline \multicolumn{4}{|c|}{ Change in ICIQ vs scores } \\
\hline & VS Score & SM Score & Q0L Score \\
\hline Difference of mean & $-21.2^{*}$ & $-37.4^{*}$ & $-7.9 *$ \\
\hline Median & -21 & -40 & -8 \\
\hline Range & -6 to -33 & -22 to -57 & -5 to- 10 \\
\hline \multicolumn{4}{|c|}{$\begin{array}{l}\text { ICIQ: Incontinence questionnaire; VS: Vaginal Symptoms; QOL: Quality } \\
\text { of Life Questionnaire }\end{array}$} \\
\hline
\end{tabular}

complication, quicker recovery and less expensive. But SSF is not appropriate in a sexually active woman and in those having a shortened vagina. In a Cochrane review, ASCP was found to be associated with a lower rate of recurrent vault prolapse compared to the vaginal SSF. ${ }^{5}$ The reported success rate with ASCP is $78 \%-100 \% .^{10}$ The present study of 41 patients has also shown a 100\% subjective and objective success rate over a the 24 months follow up period.

Newer surgical approaches like pectopexy where the vaginal vault is attached bilaterally to the iliopectineal ligaments, is advantaged by improved defecation scores over the sacrocolpopexy. ${ }^{11}$

Although laparoscopic route of sacrocolpopexy is advantaged by less operative blood loss, shorter hospital-stay and a quicker return to daily activity, the operative time is longer than open sacral colpopexy. ${ }^{12}$ Some studies have however reported similar operating times..$^{13}$ As the overall complication rate is not significantly different between open or laparoscopic SCP, the evidence is inconclusive for the choice of the most appropriate procedure. $^{14}$

No life-threatening events occurred in any of the study participants in this study and the major intraoperative complications in this study were operative hemorrhage (1/41) due to presacral vessel injury and bladder injury during dissection from vault (1/41). Immediate severe postoperative complication was postoperative febrile episodes in $34 \%$ of patients. $14.6 \%$ of patients had surgical site infection (SSI) of abdominal wound dehiscence requiring secondary surgical closure of abdominal wound. Postoperative ileus was noted in a single (2.4\%) patient who responded to conservative treatment.

At the six weeks follow up, constipation (11.7\%) and vaginitis (2.4\%) were reported by patients, which responded to medications. De novo stress urinary incontinence which has been reported to occur postoperatively in many studies was not encountered in our group of patients. Dyspareunia (9.7\%) was the only complication at $6^{\text {th }}-12^{\text {th }}$ months follow up of patients in cases 
where pelvic floor repair was done pointing to perineorrhaphy as the cause rather than mesh placement.

Vaginal bleeding, discharge and pain after SCP can be due to mesh erosion and patients should be counselled to report immediately at discharge from hospital and follow-up visits. Mesh exposure is a complication with both open and laparoscopic SCP which has been reported between $2 \%$ and $10 \%$ out of which $10 \%$ may require mesh removal. ${ }^{2}$ In our cohort of patients, there were no cases of mesh related complications for the entire period of follow up. However, long term follow-up is warranted as there is an ongoing risk of mesh related events. A newer method of mesh surgery follow-up of 32 patients by transperineal $4 \mathrm{D}$ ultrasound has been reported, where no mesh erosions were reported. ${ }^{15}$ ASCP effectiveness should always be balanced with long-term risks of mesh or suture erosion.

In the present study, the sacrohysteropexy cases experienced a $100 \%$ objective and subjective success rates. The mesh was fixed only to the posterior cervical-uterine junction for the caudal attachment and has been previously reported to be safe. ${ }^{16}$ For those who have a recurrence of apical prolapse, sacrospinous hysteropexy can be performed as it has been found to be noninferior to vaginal hysterectomy with uterosacral suspension. ${ }^{17}$ However, Maher et al.'s ${ }^{14}$ Cochrane review has not concluded clearly in favor of either uterine preserving surgery or vaginal hysterectomy for uterine prolapse.

Nair et al. ${ }^{18}$ in their review comprising 660 women from their included list of 16 studies on laparoscopic hysteropexy, reported only six pregnancies and five deliveries following the procedure. None of these patients had a recurrence of the prolapse after delivery. ${ }^{18}$ In the present study of 9/41 sacrohysteropexy, only two desired childbearing. One conceived spontaneously and delivered a healthy term baby by cesarean section. The mesh was not discernible retroperitoneally during cesarean section and the uterus was well supported post-delivery even after 6 months of follow up.

Transvaginal procedures using vaginal mesh had been introduced for hysteropexy but most are complex procedures, require specialized training and lacking much evidence in favor. They avoid the abdominal incision associated complications and are sufficient to support the vaginal apex. ${ }^{19}$ However, currently, they lack Food and Drug Administration approval.

In the present study, no vaginal bulge symptoms were reported by any patient following the procedure and a marked improvement in subjective scores was reported by all participants. Women who were sexually active before surgery remained so after surgery with improved sexual scores. There was an overall improvement in the QOL of all participants in this study.
As this study is of a single centre, the number of cases are limited, belong to a particular area and only a two year follow up has been done till now. As shown by few long-term studies, mesh related complications and recurrence of prolapse are best assessed on extended follow-up. The study participants are on an annual follow up plan and will be evaluated for surgical success and subjective improvement after abdominal mesh surgery in coming years over a long-term basis.

\section{CONCLUSION}

ASCP and sacro-hysteropexy are relatively easy to perform procedures with acceptable and good anatomical success rates. They provide optimum apical support as well as a good functional outcome and high cure rates for patients of apical vaginal prolapse. There is a significant overall improvement in vaginal symptoms, sexual function, and quality of life of women with POP undergoing these procedures and this advantage should be considered when offering these procedures.

\section{Contributions}

Surgical and Medical Practices: M.A., Mu.A., S.K., A.P., Concept: M.A., Design: M.A., A.S., Data Collection or Processing: M.A., A.S., S.G., Mu.A., S.K., A.P., Analysis or Interpretation: M.A., A.S., S.G., Literature Search: M.A., A.S., S.G., Mu.A., S.K., A.P., Writing: M.A.

\section{Ethics}

Ethics Committee Approval: Institutional Ethics Committee AlIMS Patna (IEC/AIIMS PAT/ no: 92-2016 date: 22/8/2016).

Informed Consent: A written informed consent form was obtained from the patients.

Peer-review: Externally peer-reviewed.

\section{DISCLOSURES}

Conflict of Interest: No conflict of interest was declared by the authors.

Financial Disclosure: The authors declared that this study received no financial support.

\section{REFERENCES}

1. Walter JE; UROGYNAECOLOGY COMMITTEE. Transvaginal mesh procedures for pelvic organ prolapse. J Obstet Gynaecol Can 2011; 33: 168-74.

2. Cundiff GW, Varner E, Visco AG, et al. Risk factors for mesh/suture erosion following sacral colpopexy. Am J Obstet Gynecol 2008; 199: 688.e1-5.

3. Haylen BT, Maher CF, Barber MD, et al. An International Urogynecological Association (IUGA) / International Continence Society (ICS) Joint Report on the Terminology for Female Pelvic Organ Prolapse (POP). Neurourol Urodyn 2016; 35: 137-68. 
4. Uzoma A, Farag KA. Vaginal vault prolapse. Obstet Gynecol Int 2009; 2009: 275621.

5. Maher C, Feiner B, Baessler K, Adams EJ, Hagen S, Glazener CM. Surgical management of pelvic organ prolapse in women. Cochrane Database Syst Rev. 2010; (4): CD004014.

6. Walters MD, Ridgeway BM. Surgical treatment of vaginal apex prolapse. Obstet Gynecol 2013; 121: 354-74.

7. Tyagi V, Hawthorn R, Guerrero K. Sacrocolpopexy (SCP) - A cohort study looking at short, medium and long term outcome. Med Surg Urol 2013; 2: 1-4.

8. Lane FE. Repair of posthysterectomy vaginal-vault prolapse. Obstet Gynecol 1962; 20: 72-7.

9. Post-Hysterectomy Vaginal Vault Prolapse. Green-top Guideline No. 46. RCOG/BSUG Joint Guideline. Royal College of Obstetricians and Gynaecologists; Jul, 2015.

10. Nygaard I, Brubaker L, Zyczynski HM, et al. Long-term outcomes following abdominal sacrocolpopexy for pelvic organ prolapse. JAMA 2013; 309: 2016-24.

11. Noé KG, Schiermeier S, Alkatout I, Anapolski M. Laparoscopic pectopexy: a prospective, randomized, comparative clinical trial of standard laparoscopic sacral colpocervicopexy with the new laparoscopic pectopexy-postoperative results and intermediateterm follow-up in a pilot study. J Endourol 2015; 29: 210-5.

12. ACOG Committee on Practice Bulletins--Gynecology. ACOG Practice Bulletin No. 85: Pelvic organ prolapse. Obstet Gynecol 2007; 110: 717-29.
13. Coolen AL, van Oudheusden AM, van Eijndhoven HW, van der Heijden TP, Stokmans RA, Mol BW, Bongers MY. A Comparison of Complications between Open Abdominal Sacrocolpopexy and Laparoscopic Sacrocolpopexy for the Treatment of Vault Prolapse. Obstet Gynecol Int 2013; 2013: 528636.

14. Maher C, Feiner B, Baessler K, Christmann-Schmid C, Haya N, Brown J. Surgery for women with apical vaginal prolapse. Cochrane Database Syst Rev 2016; 10: CD012376.

15. Eisenberg VH, Steinberg M, Weiner Z, Schiff E, Lowenstein L. Longterm follow-up of sacrocolpopexy mesh implants at two time intervals at least 1 year apart using 4D transperineal ultrasound. Ultrasound Obstet Gynecol 2017;49:398-403.

16. Khan A, Jaleel R, Nasrullah FD. Sacrohysteropexy performed as uterus conserving surgery for pelvic organ prolapse: Review of case files. Pak J Med Sci 2016; 32: 1174-8.

17. Detollenaere RJ, den Boon J, Stekelenburg J, et al. Sacrospinous hysteropexy versus vaginal hysterectomy with suspension of the uterosacral ligaments in women with uterine prolapse stage 2 or higher: multicentre randomised non-inferiority trial. BMJ 2015; 351: h3717.

18. Nair R, Nikolopoulos KI, Claydon LS. Clinical outcomes in women undergoing laparoscopic hysteropexy: A systematic review. Eur J Obstet Gynecol Reprod Biol 2017; 208: 71-80.

19. Kong MK, Bai SW. Surgical treatments for vaginal apical prolapse. Obstet Gynecol Sci 2016; 59: 253-60. 